\title{
Novel miR-1958 Promotes Mycobacterium tuberculosis Survival in RAW264.7 Cells by Inhibiting Autophagy Via Atg5 ${ }^{\text {s }}$
}

\author{
Shuqin Ding ${ }^{1,2 \dagger}$, Yuliang $\mathrm{Qu}^{2,3,5 \dagger}$, Shaoqi Yang ${ }^{4}, \mathrm{Ya}^{\prime} \mathrm{Z} \mathrm{Zhao}^{1 *}$, and Guangxian $\mathrm{Xu}^{2,5 *}$ \\ ${ }^{1}$ School of Basic Medical Sciences, Xi'an Jiaotong University, Xi'an, 710000, P.R. China \\ ${ }^{2}$ Department of Medical Laboratory, School of Clinical Medicine, Ningxia Medical University, Yinchuan, 750004, P.R. China \\ ${ }^{3}$ Department of Clinical Medicine, School of Clinical Medicine, Ningxia Medical University, Yinchuan, 750004, P.R. China \\ ${ }^{4}$ Department of Digestive Disease, General Hospital of Ningxia Medical University, Yinchuan, 750004, P.R. China \\ ${ }^{5}$ Ningxia Key Laboratory of Clinical and Pathogenic Microbiology, General Hospital of Ningxia Medical University, Yinchuan, 750004, P.R. China
}

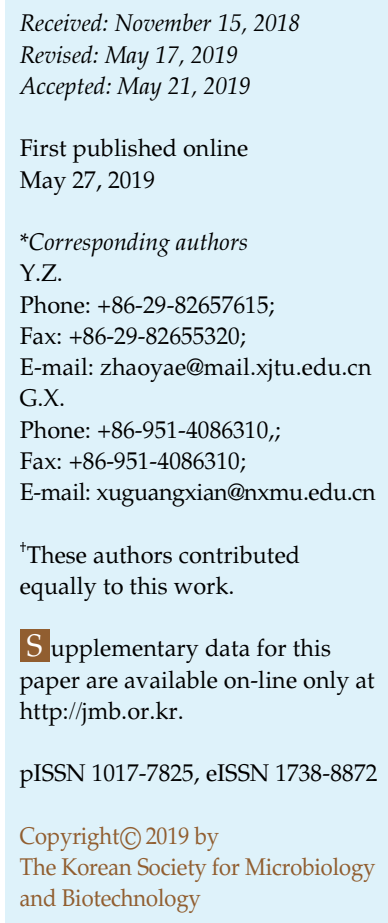

Autophagy is crucial for immune defense against Mycobacterium tuberculosis (Mtb) infection. Mtb can evade host immune attack and survival within macrophages by manipulating the autophagic process. MicroRNAs (miRNAs) are small, non-coding RNAs that are involved in regulating vital genes during $\mathrm{Mtb}$ infection. The precise role of miRNAs in autophagy with the exits of Mtb remains largely unknown. In this study, we found miR-1958, a new miRNA that could regulate autophagy by interacting with 3'UTR of autophagy-related gene 5 (Atg5). In addition, Mtb infection triggered miR-1958 expression in RAW264.7 cells. What's more, miR1958 overexpression blocked autophagic flux by impairing the fusion of autophagosomes and lysosomes. Overexpression of miR-1958 reduced Atg5 expression and LC3 puncta while inhibition of miR-1958 brought an increase of Atg5 and LC3 puncta; the opposite results were observed in detection of p62. The survival of Mtb in RAW264.7 cells transfected with mimic of miR-1958 was enhanced. Taken together, our research demonstrated that a novel miR-1958 could inhibit autophagy by interacting with Atg 5 and favored intracellular Mtb survival in RAW264.7 cells.

Keywords: miR-1958, Atg5, autophagy, bioinformatics, Mtb

\section{Introduction}

Mycobacterium tuberculosis (Mtb) is the causative agent of tuberculosis (TB) which leads to 2 million deaths each year around the world. Increasing research shows that the ability of Mtb to evade host immunologic response and is the main cause of latent infection [1]. Under normal circumstances, autophagy contributes to the degradation of intracellular bacteria, however, recent research has revealed that Mtb may hijack autophagosomes for survival by getting a power supply and even reconstructing host lipid metabolism $[2,3]$. Mtb is one of the toughest pathogens in history and relies on the characteristics of latent infection and intracellular survival, as well as emerging drug resistance [3-5]. Macrophages are the first line of defense for innate immunity against $\mathrm{Mtb}$ infection; due to specific characteristics of different strains, macrophages may become a refuge for Mtb [6]. Evidence shows that Mtb can inhibit RAB7 (a late endosomal GTPase) recruitment to affect the autophagic process in macrophages [7]. To this day, tuberculosis is still a threat to public health. Therefore, it is of great significance to explore the mechanism behind Mtb's immunity evasion.

Autophagy is a crucial biologic process which contributes to maintaining intracellular homeostasis and preventing damage caused by pathogens. There are two major steps in 
the autophagic process: formation of autophagosomes and maturation of autolysosomes [8]. Autophagy has a variety of functions that can not only resist the invasion of pathogens but also regulate the metabolism of cells themselves. For instance, the interaction between mTOR and autophagy is governed by many proteins which are responsible for sensing nutrients at lysosomal membranes [9]. Autophagy is somewhat like a central station of energy metabolism; it maintains energy balance through degradation of energy reserves such as lipids, glycogen, and proteins in the setting of increased energy demands [10]. Autophagy may be harmful or beneficial to individuals depending on its degree or magnitude [11, 12]. Moreover, numerous results reveal that microRNAs (miRNAs) are involved in regulation of autophagy [13].

MiRNAs are small non-coding RNAs and almost 21 to 25 nucleotides in length. Researchers have proved that miRNAs play important roles in controlling mRNA translation or regulating degradation of mRNA [14]. MicroRNAs exhibit multiple roles in biological processes, such as initiators, suppressors, biomarkers or even therapeutic targets [1517]. It has been proved that miR-30a involves in inhibiting autophagy by targeting BECN1 in LECs in human diabetic cataract [18]. Three miRNAs (miR-361-5p, miR-889 and $\mathrm{miR}-576-3 \mathrm{p}$ ) are used for distinguishing tuberculosis patients from healthy controls and other microbial infection, which implies that these miRNAs have great potential to serve as biomarkers for early diagnosis of tuberculosis [19]. Besides, TGF- $\beta 1$-mediated activation of the $\mathrm{miR}-20 \mathrm{a} / \mathrm{Smad} 7 / \beta$ catenin axis plays a vital role in the pathogenesis and worse prognosis of GBCs, which means miR-20a might be a potential therapeutic target in the future [20]. Moreover, miR-708 acts as a tumor suppressor by negatively regulating the anti-apoptotic protein c-FLIPL and the sensitivity of renal cancer cells [21]. These results indicate that the discovery of novel regulatory miRNAs is still of great necessity.

Many differential expression miRNAs were found through RNA sequencing in our previous research [22]. In this study, we screened a novel miR-1958 which is highly associated with regulation of autophagy. Further experiments showed that miR-1958 could inhibit autophagy by interacting with Atg5, a pivotal gene of autophagy, and thus promoted survival of intracellular Mtb in RAW264.7 cells.

\section{Material and Methods}

\section{RNA Sequencing and Bioinformatic Analysis of miRNAs}

RAW264.7 cells were treated with 3-methyladenine (3-MA, $5 \mathrm{mM}, 12 \mathrm{~h}$ ) or rapamycin $(50 \mathrm{~nm}, 2 \mathrm{~h})$, after that, total RNA was isolated with TRIzol reagent (Sigma). The target genes of miRNAs were obtained by miRWalk 3.0 (http://129.206.7.150/), miRDB (http://www.mirdb.org/miRDB/) and Targetscan (http://www. targetscan.org/). GO enrichment and KEGG pathway analysis were conducted using the Database for Annotation, Visualization and Integrated Discovery (DAVID, https://www.david.ncifcrf.gov/).

\section{Cell Culture and Transfection}

Murine macrophage RAW264.7 cells (ATCC; TIB-71) were purchased from the Type Culture Collection of the Chinese Academy of Sciences (China). The cells were grown in DMEM containing 10\% Fetal Bovine Serum (FBS) and incubated at $37^{\circ} \mathrm{C}$ with $5 \% \mathrm{CO}_{2}$. RAW264.7 cells were transfected with $50 \mathrm{nM}$ control or mimic of miR-1958 (Gene Pharma, China); $50 \mathrm{nM}$ control or $150 \mathrm{nM}$ inhibitor of miR-1958 (Gene Pharma); or $50 \mathrm{pM}$ Atg5 siRNA, by using Lipofectamine 3000 (Invitrogen, USA) according to the official manual.

\section{Bacterial Strains and CFU Assays}

H37Ra strain was purchased from the Center for Disease Control and Prevention of China. Bacilli were cultivated in Middlebrook $7 \mathrm{H} 9$ with $10 \%$ albumin dextrose catalase (ADC) supplement at $37^{\circ} \mathrm{C}$ for three weeks. The H37Ra were collected by centrifugation at $500 \times g$ for $8 \mathrm{~min}$, and resuspended with culture medium for infection. RAW264.7 cells were infected at MOI $=10$ for six hours and then washed by PBS three times to remove extracellular Mtb. For colony-forming unit (CFU) assays, RAW264.7 cells were transfected with mimic or inhibitor of miR-1958 for $24 \mathrm{~h}$ and then infected by the H37Ra bacilli suspension for $6 \mathrm{~h}$, and the extracellular H37Ra were removed by washing with PBS. The infected cells were incubated at $37^{\circ} \mathrm{C}$ for another $24 \mathrm{~h}$ and lysed by cell lysis buffer. After that, quantitative culturing was performed with 10 -fold serial dilutions on 7H10 agar plated with OADC. The plates were incubated at $37^{\circ} \mathrm{C}$ for 3 weeks, and colonies were counted.

\section{Plasmid Construction and Luciferase Reporter Assays}

The wild-type 3'Untranslated Regions (3'UTR) of Atg5 containing the seed region of miR-1958 was acquired by PCR. The mutant 3'UTR of Atg5 was constructed via PCR based on the site-directed mutagenesis theory. The primers used in the study are listed in Table 2. Each primer contained the restriction sites of SacI or MluI. The above wild-type or mutant fragments were cloned into pMIRReport vector (Ambion), producing pMIR- Report-Atg5-WT (Atg5WT) or pMIR- Report-Atg5-Mut (Atg5-Mut). Plasmids Atg5-WT or Atg5-Mut were cotransfected with control, miR-1958 mimic or inhibitor into 293T cells with Lipofectamine3000 (Invitrogen). Furthermore, pRL-TK (Renilla luciferase, purchased from Promega) was transfected in each group at the same time to evaluate transfection efficiency. The relative activity of firefly luciferase was determined by the Dual-Luciferase Reporter Assay System (Promega) following the manufacturer's protocol after $48 \mathrm{~h}$. 


\section{Quantitative Real-Time PCR}

The expression level of miR-1958 in RAW264.7 cells was tested by qRT-PCR. Total RNA was acquired by using TRIzol reagent (Sigma). For detection, cDNA was synthesized by using a Revert Aid Fisrt Strand cDNA Synthesis Kit (Thermo Fisher Scientific) and qRT-PCR was performed by using SYBR Select Master Mix (Applied Biosystems). RNU6 (for miRNA detecting) or GADPH (used in mRNA detecting) gene was used for normalization. The PCR cycles were as follows: $95^{\circ} \mathrm{C}$ for $30 \mathrm{sec}, 40$ cycles of $95^{\circ} \mathrm{C}$ for $15 \mathrm{sec}$, followed by $54^{\circ} \mathrm{C}$ for $30 \mathrm{sec}$, and $72^{\circ} \mathrm{C}$ for $30 \mathrm{sec}$. The quantity was calculated by using a comparative $\mathrm{Ct}(\Delta \Delta \mathrm{Ct})$ method.

\section{Western Blot}

Proteins were loaded onto 12\% SDS-PAGE gels and transferred to a polyvinylidene difluoride membrane (PVDF, Millipore, USA). Membranes were blocked with 5\% BSA and then incubated with antibody of Atg5, LC3B, SQSTMI/P62, or GAPDH (purchased from Cell Signaling Technology) for 12-16 h. The membranes were washed by TBST three times before being incubated with HRP-conjugated goat anti-rabbit IgG secondary antibody for $1 \mathrm{~h}$. Afterwards, ECL reagent (Thermo Fisher Scientific) was used for immunoreactive band analysis

\section{Immunofluorescence Analysis}

The RAW264.7 cells were transfected with miR-1958 mimic, inhibitor or control for $48 \mathrm{~h}$, and then cells were fixed with $4 \%$ paraformaldehyde and $0.2 \%$ Triton X-100. Before incubation with rabbit anti-LC3 antibody and FITC-conjugated goat anti-rabbit IgG, cells were blocked by $5 \%$ bovine serum albumin, and photographs of cells were captured by an Olympus DSU spinning disk confocal microscope under a $60 \mathrm{X}$ objective lens. The mRFPGFP-LC3 plasmid was transduced into RAW264.7 cells with lentiviral previously. After isolating by flow cytometry (FCM), the positive cells were then transfected with mimic or inhibitor of miR-1958, and photos were also taken with a confocal microscope under a $60 \mathrm{X}$ objective lens. At least 10 cells were triple crosssectioned in each group and counted by ImageJ software.

\section{MRFP-GFP-LC3 Plasmid Positive RAW264.7 Cell Strain}

MRFP-GFP-LC3 plasmid was transduced into RAW264.7 cells with a lentiviral system. The positive cells were isolated by FCM and preserved properly as a strain. The cells were then transfected with miR-1958 mimic or inhibitor, and pictures of the cells were captured by confocal microscope under a $60 \mathrm{X}$ objective lens. At least 10 cells were triple cross-sectioned in each group and counted by Image J software.

\section{Statistical Analysis}

All of the results are presented as mean \pm SD of independent experiments. Statistical analysis was performed by using twotailed Student's $t$-test. Significant differences were assigned to $p$ values $<0.05,<0.01$ and $<0.001$, denoted by ${ }^{*}$, ${ }^{* *}$ and ${ }^{* * *}$, respectively.

\section{Results}

\section{Bioinformatic Analysis of miR-1958}

RAW264.7 cells were treated with 3-methyladenine (3MA) or rapamycin at indicated time and dose, and then quantitative Real-Time PCR was used to verify miR-1958 expression in these cells. The expression of miR-1958 in the rapamycin-treated group was significantly increased whereas the 3-MA group maintained normal level (Fig. 1A). MiRWalk was used to obtain target genes of miR-1958 and 10447 target genes were found (binding $p$ value $>0.8$, supplementary Table). Among these genes, those associated with autophagy were screened out and their interaction was analyzed by using the STRING database. As shown in Fig. 1B, Atg5, Atg3 and Atg16L1 were highly relevant. Meanwhile, 10447 genes were also uploaded to DAVID software to perform GO enrichment and KEGG pathway analysis. Afterwards, we screened out five GO categories (GO: 0016236 macroautophagy, GO: 0000045 autophagosome assembly, GO: 0006914 autophagy, GO: 0045087 innate

Table 1. Results of GO enrichment and KEGG pathway analysis in finding genes that involved in autophagy.

\begin{tabular}{ll}
\hline \multicolumn{1}{c}{ Category } & \multicolumn{1}{c}{ Term } \\
\hline GOTERM_BP_DIRECT & GO:0016236 macroautophagy \\
GOTERM_BP_DIRECT & GO:0000045 autophagosome assembly \\
GOTERM_BP_DIRECT & GO:0006914 autophagy \\
GOTERM_BP_DIRECT & GO:0045087 innate immune response \\
GOTERM_CC_DIRECT & GO:0005737 cytoplasm \\
KEGG_PATHWAY & mmu04151:PI3K-Akt signaling pathway \\
KEGG_PATHWAY & mmu04150:mTOR signaling pathway \\
KEGG_PATHWAY & mmu04142:Lysosome \\
KEGG_PATHWAY & mmu04145:Phagosome \\
KEGG_PATHWAY & 34 \\
KEGG_PATHWAY & mm05152:Tuberculosis \\
74 \\
79
\end{tabular}


A
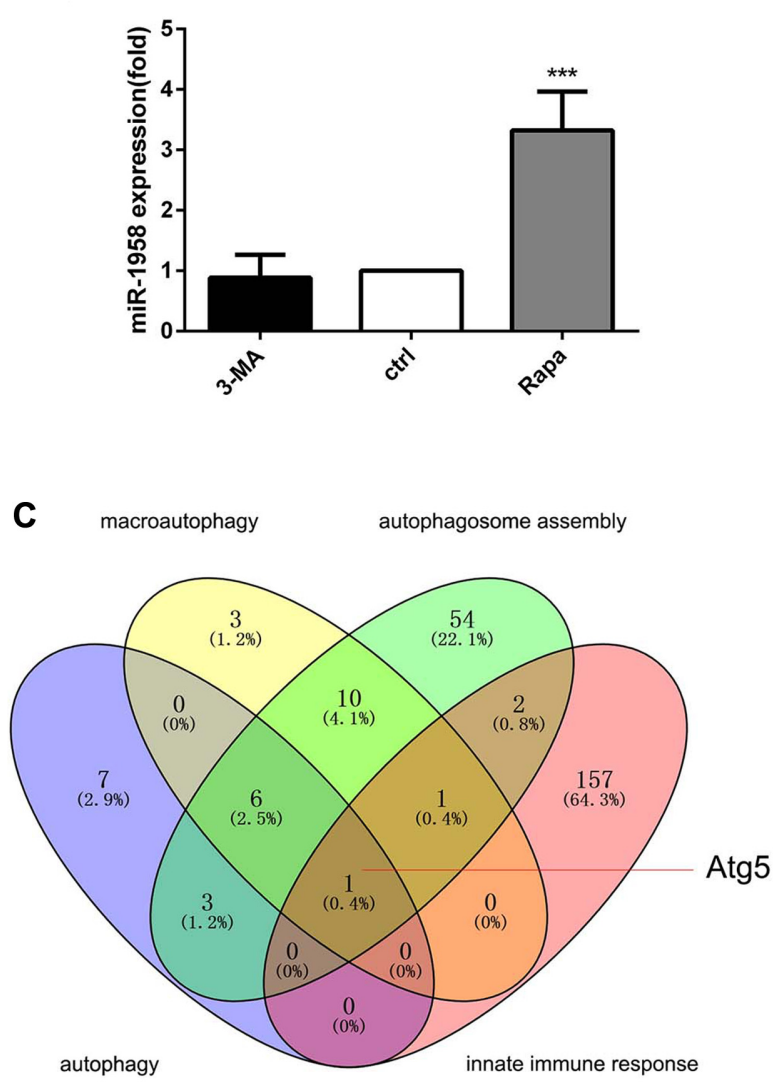

B

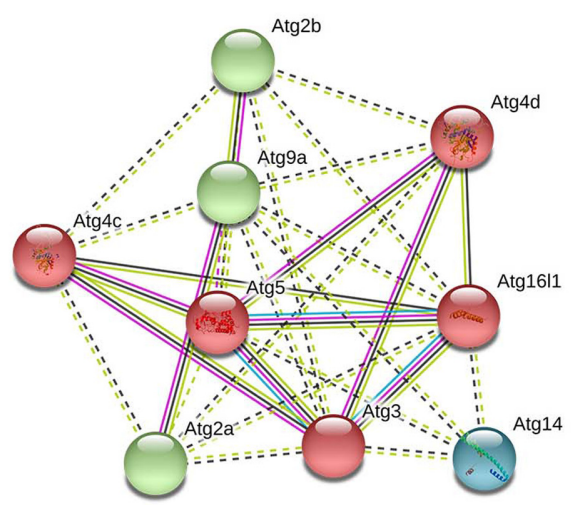

D

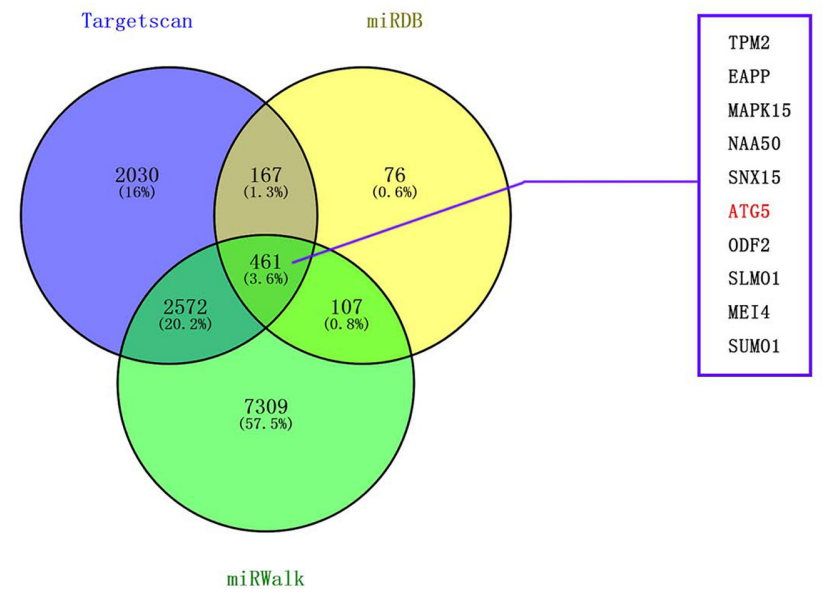

Fig. 1. Bioinformatic analysis of miR-1958.

(A) RAW264.7 cells were treated with 3-MA ( $5 \mathrm{mM}, 12 \mathrm{~h}$ ) or rapamycin $(50 \mathrm{~nm}, 2 \mathrm{~h})$, miR-1958 expression level was measured by qRT-PCR. (B) Interaction of proteins which were highly related to autophagy. (C) Intersection of miR-1958 target genes gained from four GO categories based on the results of Table 1. (D) The overlap of three different databases revealed top ten target genes of miR-1958. Data were presented by the mean \pm SD from three experiments. ${ }^{*} p<0.05,{ }^{* *} p<0.01$.

immune response, GO: 0005737 cytoplasm) and 6 pathways (mmu04151: PI3K-Akt signaling pathway, mmu04150: mTOR signaling pathway, mmu04142: Lysosome, mmu04145: Phagosome, mmu05152: Tuberculosis, mmu04140: Regulation of autophagy) that were highly correlated with autophagy (Table 1). The only gene in the intersection of five GO enrichment categories was Atg5 (Fig. 1C). Furthermore, Targetscan and miRDB were also recruited to examine the accuracy of MiRWalk. The top ten genes (ranked by scores in each database) in the intersection of the above three databases were shown in Fig. 1D. Subsequently, Atg5 was also on that list. Thus, miR-1958 and its target gene Atg5 were chosen for additional researches in our study.

\section{MiR-1958 Interacted with 3'UTR of Atg5}

Through bioinformatic analysis, 3'Untranslated Regions (3'UTR) of Atg5 which contained miR-1958 response elements was obtained (Fig. 2A). To validate whether Atg5 was the direct target of miR-1958, dual-luciferase report assays was performed, and results displayed that overexpression of miR-1958 depressed luciferase activity in 293T cells containing Atg5-WT vector whereas no change was observed in cells transfected with the Atg5-Mut vector; opposite data were acquired when inhibitor of miR-1958 was transfected into cells. However, no impacts on luciferase activity were detected in cells containing Atg5Mut vector along with miR-1958 mimic or inhibitor (Fig. 2B). These results suggested that miR-1958 could directly interact with 3'UTR of Atg5.

\section{H37Ra Infection Induced Expression of miR-1958}

RAW264.7 cells were infected with H37Ra at an MOI of 10 for $6 \mathrm{~h}$ and expression of miR-1958 was measured by qRT-PCR. The level of miR-1958 rose continuously and 
A

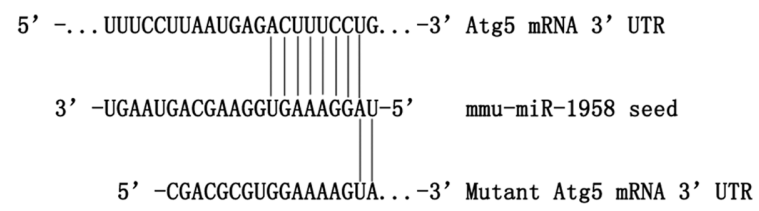

B

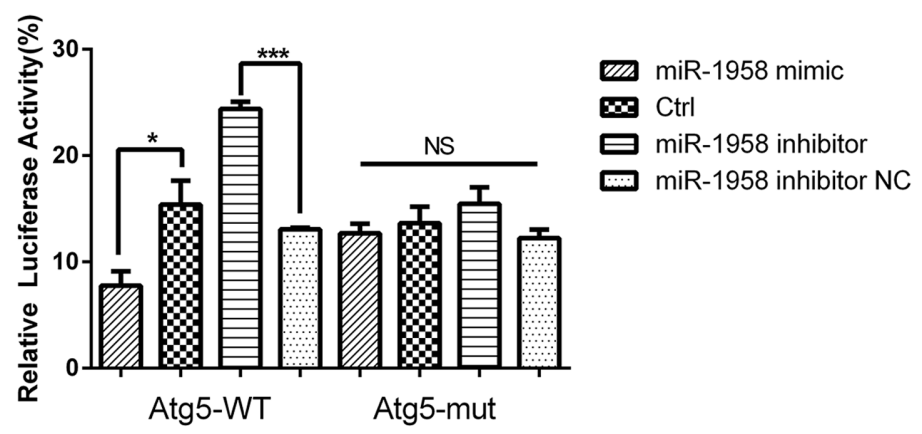

Fig. 2. MiR-1958 reduced expression of Atg5 by interacting with its 3'UTR.

(A) The seed sequence of miR-1958 and 3'UTR of Atg5 acquired from bioinformatic analysis. (B) 293T cells were transfected with control, inhibitor control, mimic of miR-1958 and wild type or mutant of Atg5 luciferease reporter plasmid, inhibitor of miR-1958 and wild type or mutant of Atg5 luciferease reporter plasmid. After $48 \mathrm{~h}$ post transfection, the luciferease activity was detected. Data were presented as mean \pm SD from three experiments. ${ }^{*} p<0.05,{ }^{* * *} p<0.001$.

reached the peak at $24 \mathrm{~h}$, after which it began to fall (Fig. 3). These data demonstrated that miR-1958 might play a role in early infection of Mtb.

\section{MiR-1958 Inhibited Autophagy by Reducing Expression} of $\operatorname{Atg} 5$

For further study, RAW264.7 cells were transfected with mimic or inhibitor of miR-1958 and mRNA expression level of Atg5 was tested by qRT-PCR. The data demonstrated that the expression of miR-1958 mRNA was significantly improved in RAW264.7 cells transfected with inhibitor of miR-1958 and declined with mimic of miR-1958 (Fig. 4A). In addition, transfection of miR-1958 mimic promoted the expression of Atg5 protein in RAW264.7 cells; in contrast, transfection of miR-1958 inhibitor resulted in the promotion of Atg5; besides, LC3-II/I ratio was suppressed in cells that

Table 2. Primer information.

\begin{tabular}{|c|c|}
\hline Primers & Sequence $\left(5^{\prime}-3^{\prime}\right)$ \\
\hline \multirow[t]{3}{*}{ miR-1958 } & RT Stem-loop: CTCAACTGGTGTCGTGGAGTCGGCAATTCAGTTGAGACTTACTG \\
\hline & Forward: ACACTCCAGCTGGGTAGGAAAGTGGAAGCA \\
\hline & Reverse: TGGTGTCGTGGAGTCG \\
\hline \multirow[t]{2}{*}{ U6 } & Forward: CTCGCTTCGGCAGCACA \\
\hline & Reverse: AACGCTTCACGAATTTGCGT \\
\hline \multirow[t]{2}{*}{ Negetive control } & Forward: UUCUCCGAACGUGUCACGUTT \\
\hline & Reverse: ACGUGACACGUUCGGAGAATT \\
\hline \multirow[t]{2}{*}{ Mir-1958 mimics } & UAGGAAAGUGGAAGCAGUAAGU \\
\hline & UUACUGCUUCCACUUUCCUAUU \\
\hline Mir-1958 inhibitor & ACUUACUGCUUCCACUUUCCUA \\
\hline Atg5 mRNA & Forward: GTGGAGGCAACAGAAACC \\
\hline \multirow[t]{2}{*}{ Atg5-WT } & Forward: CCGAGCTCCAAGCCAAGGAGGAGAAGAT \\
\hline & Reverse: CGACGCGTACTGGAAAGGAACGGGAGAT \\
\hline \multirow[t]{2}{*}{ Atg5-Mut } & Forward: CCGAGCTCCAAGCCAAGGAGGAGAAGAT \\
\hline & Reverse: CGACGCGTGAAAAGGATGGGTTTACTGTAATTTGA \\
\hline \multirow[t]{2}{*}{ GADPH } & Forward: GCACCGTCAAGGCTGAGAAC \\
\hline & Reverse: TGGTGAAGACGCCAGTGGA \\
\hline
\end{tabular}




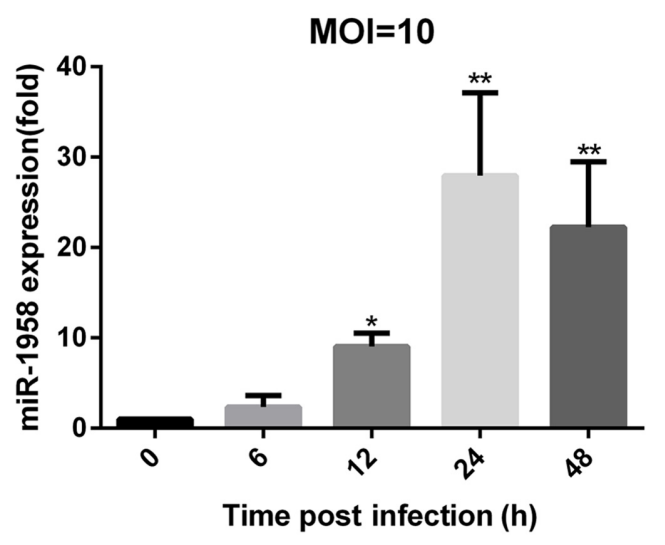

Fig. 3. H37Ra infection induced expression of miR-1958. RAW264.7 cells were infected by H37Ra at MOI of 10 for $6 \mathrm{H}$ and total RNA were isolated at indicate time for measuring the expression of miR-1958 by using qRT-PCR. Data were presented as mean \pm SD from three experiments. ${ }^{*} p<0.05,{ }^{* *} p<0.01,{ }^{* * *} p<0.001$.

were transfected with miR-1958 mimic, inhibitor of miR1958 did exactly opposite (Fig. 4B). Moreover, the expression of p62 and LC3 in RAW264.7 cells were further detected and the results revealed that miR-1958 overexpression

A

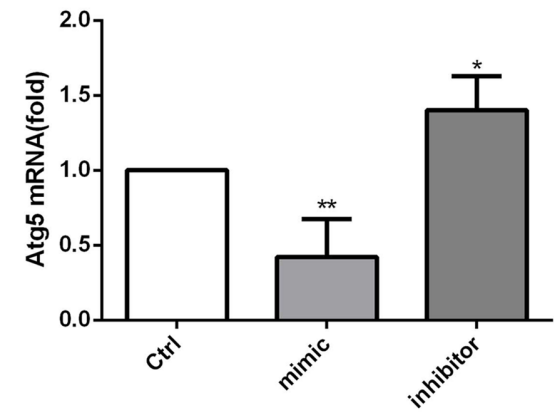

C

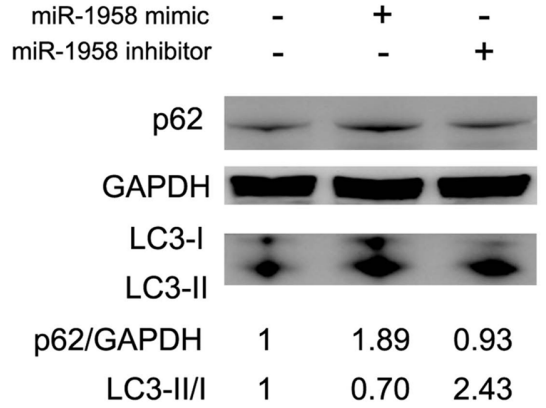

leads to decrease of LC3-II/I ratio and accumulation of p62, and vice versa (Fig. 4C). Atg5 siRNA was transfected into RAW264.7 cells with miR-1958 mimic or inhibitor, as a result, the protein of Atg5 and LC3 was significantly declined by siRNA; mimic of miR-1958 strengthened the ability of siRNA while inhibitor of miR-1958 failed to convert the expression of Atg5 and both of LC3-I and LC3II (Fig. 4D). For immunofluorescence analysis, RAW264.7 cells were transfected with mimic or inhibitor of miR-1958 for $24 \mathrm{~h}$ and then treated with rapamycin for $2 \mathrm{~h}$. The pictures were captured by confocal microscope under a $60 \mathrm{X}$ objective lens; the puncta of LC3 were significantly restrained in the mimic group while inhibitor of miR-1958 could elevate the quantity of LC3 puncta (Fig. 5A). The count of LC3 puncta performed by ImageJ also confirmed these consequences (Fig. 5B). Taken together, these results indicated that miR-1958 impaired autophagy posttranscriptionally via Atg5.

\section{MiR-1958 Suppressed Autophagic Flux and Favored H37Ra Survival in RAW264.7}

The GFP-RFP-LC3 positive RAW264.7 cells were constructed and then further transfected with miR-1958 mimic or

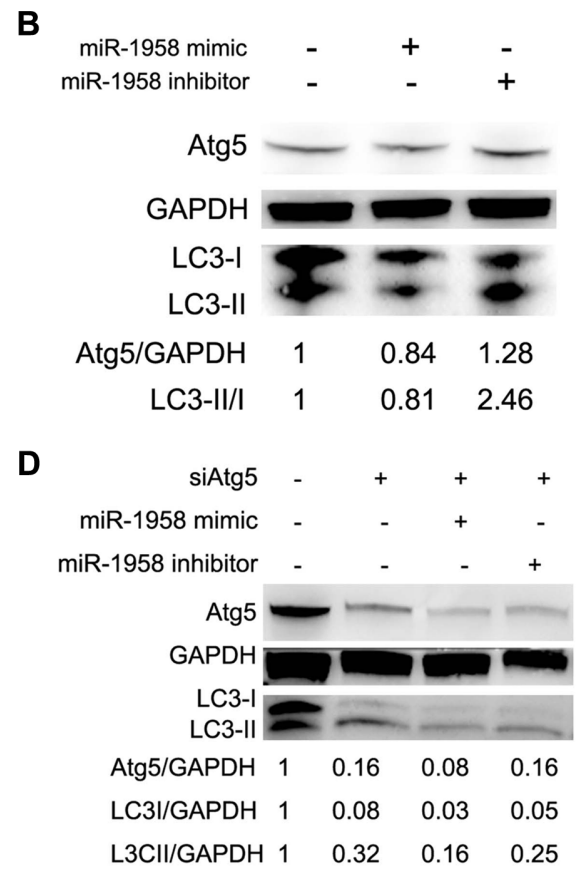

Fig. 4. MiR-1958 inhibited autophagy by reducing expression of Atg5.

(A) RAW264.7 cells were transfected with mimic or inhibitor of miR-1958 for $48 \mathrm{~h}$. The expression of Atg5 mRNA was detected by qRT-PCR. (B) RAW264.7 cells were transfected with mimic or inhibitor of miR-1958. After $48 \mathrm{~h}$, the quantities of Atg5 and LC3-I/II were detected by western blot. GAPDH was used as internal control. (C) RAW264.7 cells were transfected with mimic or inhibitor of miR-1958. After 48 h, the quantities of p62 and LC3-I/II were detected by western blot. (D) RAW264.7 cells were transfected with siRNA of Atg5 along with mimic of inhibitor of miR1958 for $48 \mathrm{~h}$. The protein level was determined by western blot and analyzed by ImageJ software. 

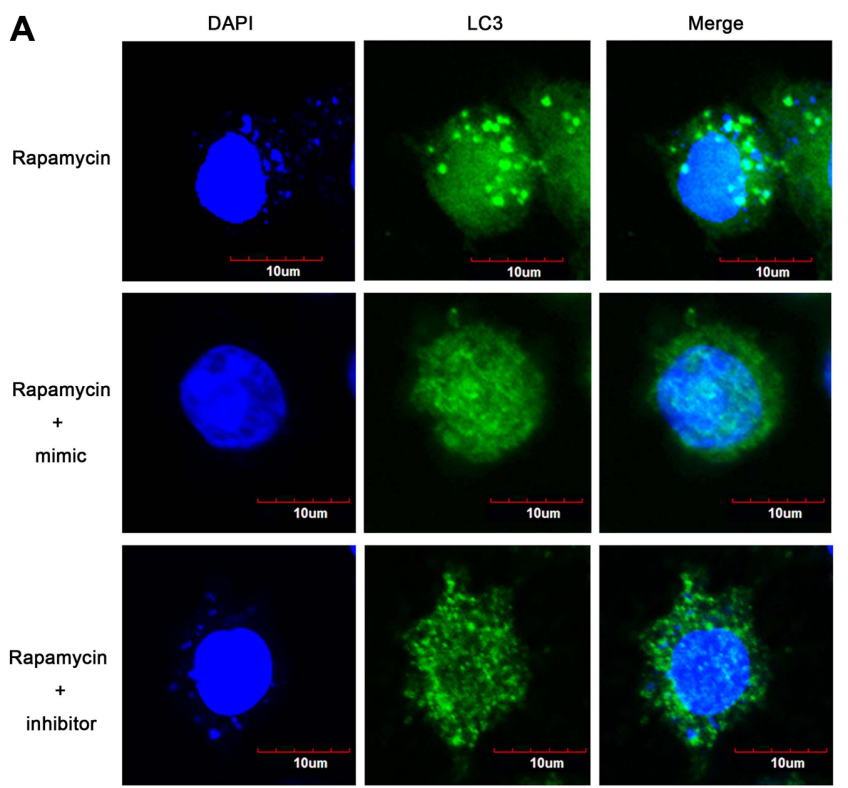

B

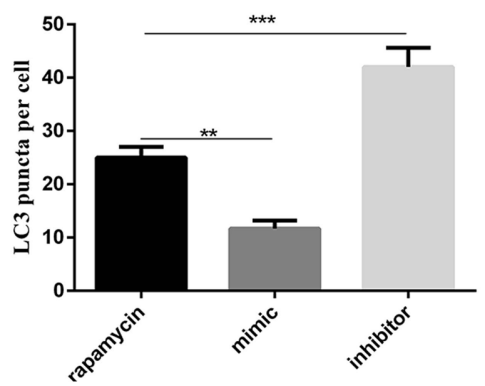

Fig. 5. MiR-1958 impaired autophagy and confirmed by immunofluorescence analysis.

(A) The RAW264.7 cells were transfected with control, mimic or inhibitor of miR-1958. After $48 \mathrm{~h}$, the cells were treated with rapamycin for $2 \mathrm{~h}$ and then fixed and incubated with rabbit anti-LC3 antibody, followed by FITC-conjugated goat anti-rabbit IgG. LC3 puncta was detected by confocal microscopy. (B) The puncta of LC3 was counted by ImageJ software with at least three different pictures $(n>10)$. Data were presented as mean $\pm \mathrm{SD},{ }^{* *} p<0.01,{ }^{* * *} p<0.001$.

inhibitor and the pictures were acquired by using confocal microscope under a $60 \mathrm{X}$ objective lens. The red puncta had obviously vanished in the mimic group which suggested miR-1958 was a suppressor of rapamycin-induced autophagic flux and fusion of autophagosomes and lysosomes; inhibitor of miR-1958 dramatically improved appearance of red puncta, indicating autolysosomes and autophagic flux were promoted (Figs. 6A and 6B). Since autophagy plays important roles in defense against Mtb invasion, we thus performed CFU assays to validate H37Ra survival in RAW264.7 cells transfected with miR-1958 mimic or

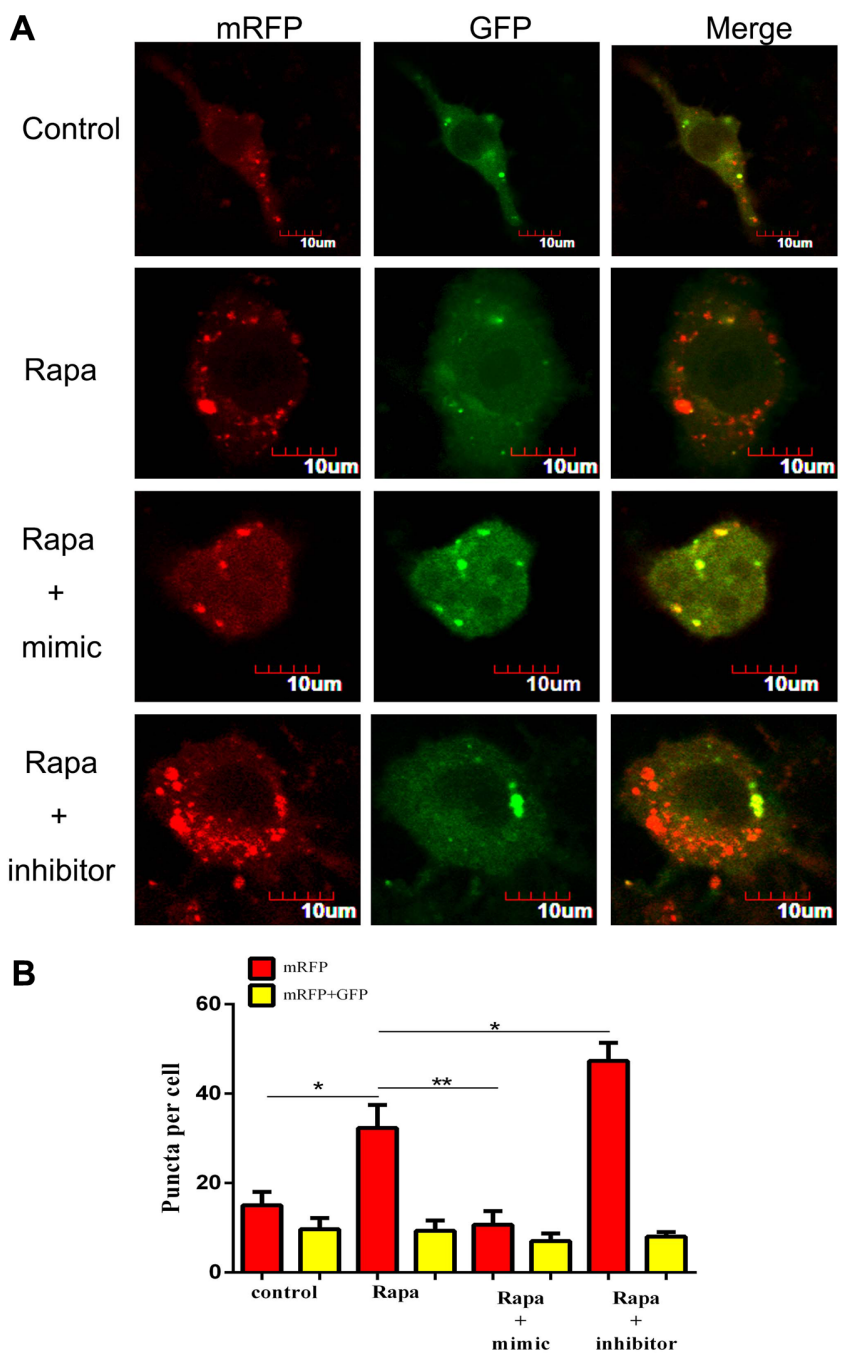

Fig. 6. MiR-1958 suppressed autophagic flux via Atg5.

(A) The RFP-GFP-LC3 positive RAW264.7 cells were transfected with control, mimic or inhibitor of miR-1958. The pictures were captured by confocal microscope under a $60 \mathrm{X}$ objective lens. (B) The puncta of red or yellow was counted by Image software with at least three different pictures $(n>10)$. Data were presented as mean \pm SD, ${ }^{* *} p<0.01, * * * p<0.001$.

inhibitor and control. The results revealed that mimic of miR-1958 significantly promoted intracellular H37Ra survival compared to rapamycin and the control group (Fig. 7). In conclusion, miR-1958 favored H37Ra survival in RAW264.7 by inhibiting autophagy via Atg5.

\section{Discussion}

Mycobacterium tuberculosis is one of the toughest known bacteria in human history. Due to its characteristics, Mtb 


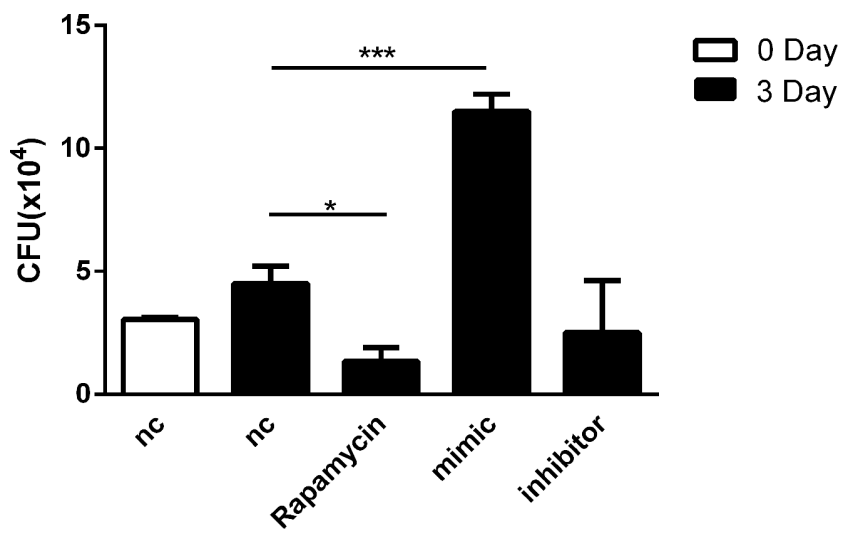

Fig. 7. MiR-1958 favored H37Ra survival in RAW264.7. RAW264.7 cells were transfected with control, mimic or inhibitor of miR-1958 for $24 \mathrm{~h}$ and then infected by H37Ra at MOI of 10 for $24 \mathrm{~h}$. Intracellular survival of H37Ra was evaluated by CFU assay. Data were presented as mean \pm SD from three experiments. ${ }^{*} p<0.05$, ${ }^{* * *} p<0.001$

can evade immunologic strike and result in persistent infection [23]. Autophagy usually plays important roles in antimicrobial responses [24] and innate defense against mycobacterium [25]. What's more, many miRNAs are found to be the regulators of autophagy [26, 27]. For instance, miR-125a and miR-315 could reduce the expression of Uvrag and led to the inhibition of autophagy in mice [28]. Another study showed that miR-182, miR-155 and miR-197 had diagnostic potential in determining patients with lung cancer, pneumonia and tuberculosis [29]. Nonetheless, the role of miRNAs in regulation of autophagy still remains largely unknown and conceals a wealth of knowledge. In this study, we found a novel miR-1958 that could inhibit autophagy and promote intracellular Mtb survival by targeting Atg5.

Bioinformatics is often utilized to find novel miRNAs. To identify the function of miRNAs, the target genes of miRNAs should be predicted first. MiRWalk 3.0 is a newly updated online tool which is able to mine multiple miRNA targets rapidly. In our study, 10447 genes were acquired as the target of miR-1958. All target genes were uploaded to DAVID, a database that allows researchers to sort gene function by annotation system [30]. After all genes were annotated, the particular function of genes can be filtered out. Five GO enrichment categories that are crucial for regulation of autophagy were selected. Notably, Atg5 was inclusive in five GO categories and five of six pathways, suggesting that Atg5 was significant for autophagy. Two more databases were used to verify the accuracy of target gene prediction of miRWalk 3.0 and the results turned out to be credible. Absolutely, the roles of miR-1958 in other biological processes need further verification.

Autophagy-related genes (ATG) are a family of genes that correlate with each other to regulate the process of autophagy. There are approximately 30 genes in the family and each gene contributes to a different stage of autophagy. For instance, ATG2, ATG5, ATG7 and ATG10 negatively affect powdery mildew resistance and mildew-induced cell death in Arabidopsis [31]. Overexpression of Atg5 extends leanness and lifespan in mice, suggesting that Atg5 is involved in promoting autophagic activity [32]. Moreover, miRNAs often participate in mediation of ATG genes. MiR$181 \mathrm{~A}$ is found to inhibit autophagic activity by interacting with ATG5 3'UTR, which proves miR-181A is critical for regulation of autophagy [27]. In our study, miR-1958 showed potential of interacting with Atg5. Luciferase activity was decreased by mimic of miR-1958 whereas inhibitor of miR-1958 caused promotion of luciferase activity in 293T cells transfected with Atg5-WT vector. The effects of miR1958 mimic and inhibitor were invalid in cells transfected with Atg5-Mutant vector. Furthermore, qRT-PCR and western bolt indicated that miR-1958 inhibited the expression of Atg 5 both in protein and mRNA levels. Since Atg 5 was crucial for autophagosome formation, the inhibition of Atg5 caused by miR-1958 undoubtedly led to mediation of autophagy. Significant decrease of Atg5 was observed in the presence of miR-1958 mimic while inhibitor of miR1958 brought an increase of Atg5.

LC3 is an autophagic marker. When autophagy is formed, cytoplasmic LC3 (LC3-I) enzymatically dissolves a small segment of polypeptide and transforms into autophagic membrane (LC3-II), which suggests LC3 plays an important role in autophagosome closure [33]. Obviously, the ratio of LC3-II/I can estimate the level of autophagy. Therefore, in our study, the changes of LC3-II/I ratio were detected by western blot, and the puncta of LC3 were measured by immunofluorescence. Each result confirmed that miR-1958 played an important role in regulation of autophagy. RAW264.7 cells were detected and the results indicated that miR-1958 could strengthen the silent ability of siRNA. Since autophagy is a dynamic process that is manipulated by diverse genes, the interaction of Atg5 and other autophagy-related genes is worthy to explore. Moreover, immunofluorescence analysis also suggested that miR-1958 mimic could reduce the autophagosomes while inhibitor of miR-1958 promoted the LC3 puncta. Considering that Mtb often protects itself by inhibiting autophagy and avoids eradication of immune system [34], RAW264.7 cells trans- 
fected with mimic or inhibitor of miR-1958 were further infected by H37Ra. Collectively, our study revealed a novel miR-1958 and demonstrated that miR-1958 could be a regulator of rapamycin-induced or Mtb-induced autophagy.

\section{Acknowledgments}

This work was supported by the National Natural Science Foundation of China (No. 81471972, No. 31472168), 2015 Ningxia Scientific and Technological Innovation Leader Training Project (KJT2015020), and First-Class Discipline Construction of Clinical Medicine of Ningxia Medical University (NXYLXK2017A05).

\section{Conflict of Interest}

The authors have no financial conflicts of interest to declare.

\section{References}

1. Korb VC, Chuturgoon AA, Moodley D. 2016. Mycobacterium tuberculosis: manipulator of protective immunity. Int. J. Mol. Sci. 17: 131.

2. Ouimet M, Koster S, Sakowski E, Ramkhelawon B, van Solingen C, Oldebeken S, et al. 2016. Mycobacterium tuberculosis induces the miR-33 locus to reprogram autophagy and host lipid metabolism. Nat. Immunol. 17: 677-686.

3. Nathan C, Barry CE. 2015. Tuberculosis drug development: immunology at the table. Immunol. Rev. 264: 308-318.

4. Ren NA, Gao G, Sun YUE, Zhang L, Wang H, Hua W, et al. 2015. MicroRNA signatures from multidrug-resistant Mycobacterium tuberculosis. Mol. Med. Report. 12: 6561-6567.

5. Shao Y, Yang D, Xu W, Lu W, Song H, Dai Y, et al. 2011. Epidemiology of anti-tuberculosis drug resistance in a chinese population: current situation and challenges ahead. BMC Public Health. 11: 110-110.

6. Guirado E, Schlesinger LS, Kaplan G. 2013. Macrophages in tuberculosis: friend or foe. Semin. Immunopathol. 35: 563-583.

7. Chandra P, Ghanwat S, Matta SK, Yadav SS, Mehta M, Siddiqui Z, et al. 2015. Mycobacterium tuberculosis inhibits RAB7 recruitment to selectively modulate autophagy flux in macrophages. Sci. Rep. 5: 16320.

8. Min Liang ZH, Kaori Sakamoto, Cao XCaG. 2017. Mycobacteria and autophagy: many questions and few answers. Curr. Issues Mol. Biol. 21: 63-71

9. Dunlop EA, Tee AR. 2014. mTOR and autophagy: A dynamic relationship governed by nutrients and energy. Semin. Cell Dev. Biol. 36: 121-129.
10. Kim KH, Lee M-S. 2014. Autophagy as a crosstalk mediator of metabolic organs in regulation of energy metabolism. Rev. Endocr. Metab. Disord. 15: 11-20.

11. Pan W-R, Chen Y-LS, Hsu H-C, Chen W-J. 2015. Antimicrobial peptide GW-H1-induced apoptosis of human gastric cancer AGS cell line is enhanced by suppression of autophagy. Mol. Cell Biochem. 400: 77-86.

12. Mortensen M, Ferguson DJP, Edelmann M, Kessler B, Morten KJ, Komatsu M, et al. 2010. Loss of autophagy in erythroid cells leads to defective removal of mitochondria and severe anemia in vivo. Proc. Natl. Acad. Sci. USA 107: 832-837.

13. Rothchild AC, Sissons JR, Shafiani S, Plaisier C, Min D, Mai D, et al. 2016. MiR-155-regulated molecular network orchestrates cell fate in the innate and adaptive immune response to Mycobacterium tuberculosis. Proc. Natl. Acad. Sci. USA 113: E6172-E6181.

14. Ameres SL, Zamore PD. 2013. Diversifying microRNA sequence and function. Nat. Rev. Mol. Cell Biol. 14: 475-488.

15. Su Z, Yang Z, Xu Y, Chen Y, Yu Q. 2015. MicroRNAs in apoptosis, autophagy and necroptosis. Oncotarget 6: 84748490.

16. Romano G, Kwong LN. 2017. Diagnostic and therapeutic applications of miRNA-based strategies to cancer immunotherapy. Cancer Metastasis Rev. 37: 45-53.

17. Wen Y, Han J, Chen J, Dong J, Xia Y, Liu J, et al. 2015. Plasma miRNAs as early biomarkers for detecting hepatocellular carcinoma. Int. J. Cancer. 137: 1679-1690.

18. Zhang L, Cheng R, Huang Y. 2017. MiR-30a inhibits BECN1-mediated autophagy in diabetic cataract. Oncotarget 8: $77360-77368$

19. Qi Y, Cui L, Ge Y, Shi Z, Zhao K, Guo X, et al. 2012. Altered serum microRNAs as biomarkers for the early diagnosis of pulmonary tuberculosis infection. BMC Infect. Dis. 12: 384384.

20. Chang Y, Liu C, Yang J, Liu G, Feng F, Tang J, et al. 2013. MiR-20a triggers metastasis of gallbladder carcinoma. J. Hepatol. 59: 518-527.

21. Kim E-A, Kim S-W, Nam J, Sung E-G, Song I-H, Kim J-Y, et al. 2016. Inhibition of c-FLIP(L) expression by miRNA-708 increases the sensitivity of renal cancer cells to anti-cancer drugs. Oncotarget 7: 31832-31846.

22. Guo L, Zhao J, Qu Y, Yin R, Gao Q, Ding S, et al. 2016. MicroRNA-20a inhibits autophagic process by targeting ATG7 and ATG16L1 and favors mycobacterial survival in macrophage cells. Front Cell Infect. Microbiol. 6: 134.

23. Pai RK, Convery M, Hamilton TA, Boom WH, Harding CV. 2003. Inhibition of IFN- $\gamma$-induced class II transactivator expression by a 19-kDa lipoprotein from mycobacterium tuberculosis: a potential mechanism for immune evasion. $J$ Immunol. 171: 175-184. 
24. Moy RH, Cherry S. 2013. Antimicrobial autophagy: a conserved innate immune response in Drosophila. J. Innate. Immun. 5: 444-455.

25. Jo EK. 2013. Autophagy as an innate defense against mycobacteria. Pathog. Dis. 67: 108-118.

26. Korkmaz G, Tekirdag KA, Ozturk DG, Kosar A, Sezerman OU, Gozuacik D. 2013. MIR376A is a regulator of starvationinduced autophagy. PLoS One 8: e82556.

27. Tekirdag KA, Korkmaz G, Ozturk DG, Agami R, Gozuacik D. 2013. MIR181A regulates starvation- and rapamycin-induced autophagy through targeting of ATG5. Autophagy 9: 374-385.

28. Kim Y, Lee J, Ryu H. 2015. Modulation of autophagy by miRNAs. BMB Rep. 48: 371-372.

29. Abd-El-Fattah AA, Sadik NAH, Shaker OG, Aboulftouh ML. 2013. Differential microRNAs expression in serum of patients with lung cancer, pulmonary tuberculosis, and pneumonia. Cell Biochem. Biophys. 67: 875-884.

30. Huang DW, Sherman BT, Tan Q, Kir J, Liu D, Bryant D, et al. 2007. DAVID Bioinformatics resources: expanded annotation database and novel algorithms to better extract biology from large gene lists. Nucleic Acids Res. 35: W169-175.

31. Wang Y, Nishimura MT, Zhao T, Tang D. 2011. ATG2, an autophagy-related protein, negatively affects powdery mildew resistance and mildew-induced cell death in Arabidopsis. Plant J. 68: 74-87.

32. Pyo J-O, Yoo S-M, Ahn H-H, Nah J, Hong S-H, Kam T-I, et al. 2013. Overexpression of Atg5 in mice activates autophagy and extends lifespan. Nat. Commun. 4: 2300.

33. Fujita N, Hayashi-Nishino $M$, Fukumoto $H$, Omori $H$, Yamamoto A, Noda T, et al. 2008. An Atg4B mutant hampers the lipidation of LC3 paralogues and causes defects in autophagosome closure. Mol. Biol. Cell. 19: 4651-4659.

34. Shin D-M, Jeon B-Y, Lee H-M, Jin HS, Yuk J-M, Song C-H, et al. 2010. Mycobacterium tuberculosis eis regulates autophagy, inflammation, and cell death through redox-dependent signaling. PLoS Pathog. 6: e1001230. 